\title{
Experiencia temprana en la aplicación de un programa ERAS en hepatectomías abiertas menores por metástasis de origen colorrectal, con resecciones simultáneas de colon o sin ellas
}

\author{
Gustavo Nari ${ }^{1,2}$, José Layún ${ }^{2}$, Lino Molina ${ }^{1}$, Luis Barrionuevo ${ }^{1}$, Eugenio CecchetTo², Sandra Rojo²
}

Palabras clave: neoplasias del colon; metástasis de la neoplasia; hepatectomía; protocolos clínicos; curva de aprendizaje; evolución clínica; evaluación de costo-efectividad.

\begin{abstract}
Resumen
Introducción. La aplicación de programas de tipo vía rápida (fast track) en cirugía abdominal mayor fue inicialmente implementada en cirugía abierta de colon con resultados comparables a los del abordaje laparoscópico. La disminución de la estancia hospitalaria $y$ de los costos hospitalarios fueron las principales ventajas. Recientemente, se trasladó la aplicación de protocolos de recuperación posquirúrgica rápida (Enhanced Recovery After Surgery, ERAS) a la cirugía hepática con resultados aparentemente alentadores.

El objetivo de este trabajo fue valorar los resultados iniciales obtenidos en un grupo de pacientes sometidos a hepatectomías menores por metástasis hepáticas, con resección simultánea de colon o sin ella.
\end{abstract}

1 Servicio de Cirugía, Hospital Tránsito Cáceres de Allende, Córdoba, Argentina

2 Sección de Oncología Digestiva, Hospital Oncológico Provincial Dr. Urrutia, Córdoba, Argentina

Fecha de recibido: 24 de abril de 2017

Fecha de aprobación: 1 de septiembre de 2017

Citar como: Nari G, Layún J, Molina L, Barrionuevo L, Cecchetto E, Rojo S. Experiencia temprana en la aplicación de un programa ERAS en hepatectomías abiertas menores por metástasis de origen colorrectal, con resecciones simultáneas de colon o sin ellas. Rev Colomb Cir. 2017;32:283-89.
Métodos. Se evaluaron los datos de 13 resecciones hepáticas menores por metástasis hepáticas de origen colorrectal con resección simultánea del tumor primario o sin ella, las cuales se incorporaron a un protocolo ERAS. Se evaluaron los datos demográficos, de las hepatectomías, de la observancia del protocolo y de algunos factores que pueden afectar los resultados de una recuperación rápida, así como también lo que se considera recuperación total al momento del egreso.

Resultados. En las 13 resecciones se pudo llevar adelante la aplicación del protocolo. Como resultado principal, se logró una corta estancia hospitalaria (3,69 días), el cumplimiento del protocolo fue alto y la recuperación total al egreso fue de $46,2 \%$.

Conclusión. La aplicación de este protocolo ha sido exitosa en cuanto a una franca disminución de la estancia hospitalaria. El número de pacientes es pequeño, pero los resultados son esperanzadores.

\section{Introducción}

Henri Kehlet fue el primero en proponer un programa multimodal de rehabilitación en cirugía del colon ${ }^{1,2}$, obteniendo resultados francamente alentadores en lo que se refiere a la recuperación posoperatoria, acortamiento de la estancia hospitalaria y, en consecuencia, de los costos. Posteriormente, varios grupos implementaron estos protocolos quirúrgicos de recuperación rápida (Enhanced Recovery After Surgery, ERAS) en cirugía 
de colon, corroborando los resultados de Kehlet ${ }^{3}$. La extensión a otras cirugías abdominales mayores fue la consecuencia lógica y se elaboraron programas ERAS para cirugía pancreática, gástrica y otras ${ }^{4-6}$.

La mayoría de los autores coinciden en afirmar que con los programas ERAS se logra una importante reducción de la estancia hospitalaria, que ronda el 50\%, y también, de la incidencia de complicaciones ${ }^{7-15}$.

Existen pocos trabajos publicados respecto a los resultados de la aplicación de programas ERAS en hepatectomías ${ }^{16}$. En algunas publicaciones de grupos con experiencia, si bien existen diferencias en los protocolos, la mayoría coincide en sus ventajas ${ }^{17}$.

El objetivo de este trabajo fue valorar los resultados obtenidos en el inicio de una experiencia de la aplicación de un programa ERAS en resecciones hepáticas menores abiertas para el tratamiento de metástasis hepáticas de origen colorrectal.

\section{Material y métodos}

Se seleccionaron los pacientes sometidos a hepatectomías menores por metástasis de origen colorrectal en el último año y medio en la Unidad de Cirugía Oncológica Digestiva del Departamento de Cirugía del Hospital Tránsito Cáceres de Allende, y se apartaron aquellos incluidos dentro del programa ERAS para hepatectomías y que fueron la población de estudio.

Se analizó de manera retrospectiva la información recolectada prospectivamente. Se evaluaron los datos demográficos, los correspondientes a las hepatectomías, la morbimortalidad, el tiempo de estancia hospitalaria y los datos correspondientes al protocolo ERAS utilizado.

Se utilizó la nomenclatura Brisbane 2000 para hepatectomías (Brisbane 2000 Nomenclature of Hepatic Anatomy and Resections) ${ }^{18}$. Las fístulas biliares se clasificaron según la propuesta del International Study Group of Liver Surgery (ISGLS) ${ }^{19} \mathrm{y}$, las complicaciones, según la de Dindo, et al. ${ }^{20}$.

El programa ERAS fue adaptado a las posibilidades de nuestro hospital y los puntos principales se describen brevemente a continuación.

\section{Programa ERAS utilizado}

\section{Preoperatorio}

- Información completa sobre el procedimiento y sus alcances, con participación del paciente en su desarrollo.

- Día preoperatorio: restricción en la dieta la noche anterior a la cirugía; ingestión de líquidos ricos en carbohidratos hasta dos horas antes de la cirugía; preparación intestinal solamente si se programa resección simultánea de colon.

\section{Intraoperatorio}

- Control de líquidos administrados, con balance cercano a cero

- $\quad$ Sin uso de drenajes

- Sonda nasogástrica que se retira al finalizar la cirugía

- Catéter epidural o uso de morfina intratecal

\section{Posoperatorio}

- Supresión de los líquidos intravenosos, dentro de las primeras 48 horas

- Inicio de dieta líquida, en las primeras 24 horas

- Inicio de dieta regular, en las primeras 48 horas

- Retiro de la sonda vesical, en las primeras 24 horas

- Inicio de deambulación, entre las 6 y 12 primeras horas del posoperatorio

\section{Estadística}

Las variables continuas se expresaron como mediana y rango. En la evaluación de la relación de las variables continuas (deambulación, pérdidas hemáticas, complicaciones y estancia hospitalaria), se utilizó la prueba de Mann-Whitney y, en las variables categóricas dicotómicas, se elaboraron tablas de contingencia y se usó el test de Fisher. Se consideró significativa una $\mathrm{p}$ menor de 0,05 . Se utilizó el paquete estadístico SPSS ${ }^{\circledR}$, versión 13 (SPSS, Inc. Chicago, IL. USA). 


\section{Resultados}

Se practicaron 13 resecciones hepáticas menores en 12 pacientes, por metástasis de cáncer colorrectal. La mayoría de los pacientes eran hombres. Ninguno presentaba alteraciones en las pruebas preoperatorias de laboratorio. Los datos demográficos se presentan en la tabla 1 .

TABLA 1.

Datos demográficos

\begin{tabular}{lc}
\hline \multicolumn{1}{c}{ Variable } & Mediana (\% o rango) \\
\hline Hombres & $9(69)$ \\
Edad & $58,77(45-79)$ \\
Alcohol & $1(7,7)$ \\
Tabaco & $4(30)$ \\
Ejercicio físico & $5(38,4)$ \\
Obesidad & $2(15,3)$ \\
IMC & $27,46(25-42)$ \\
KTR & $12(92,3)$ \\
ASA & II: $4(30)$ \\
& III: $9(70)$ \\
\hline
\end{tabular}

IMC: índice de masa corporal; KTR: kinesioterapia respiratoria; ASA: clasificación de la American Society of Anesthesiologists

Los datos de la quimioterapia, las resecciones hepáticas y las metástasis hepáticas se muestran en la tabla 2. Es de destacar que, en siete ocasiones, la resección hepática se asoció con resección simultánea del tumor primario de colon. Uno de los pacientes que reingresaron por presentar síndrome anémico, y que perdió, aproximadamente, $1.000 \mathrm{ml}$ de sangre durante la cirugía, requirió transfusión de una unidad de glóbulos rojos y se le practicó una segunda resección hepática. Las pérdidas hemáticas se detallan en la tabla 3.

En todos los pacientes, se logró una resección $\mathrm{R}_{0}$ con márgenes libres. Hubo dos (15,38 \%) complicaciones posoperatorias: una Dindo-Clavien de grado 1, consistente en una colección serosa que drenó espontáneamente y no requirió ningún tratamiento específico más allá de los cuidados domiciliarios por parte del paciente, y otra Dindo-Clavien 2, que correspondió al síndrome anémico referido anteriormente.

En lo que respecta a la evaluación de los datos del programa ERAS, se dejó drenaje intraabdominal en dos oportunidades, en los pacientes que tuvieron un sangrado superior a $600 \mathrm{ml}$ (tabla 4). La deambulación se inició,
TABLA 2.

Metástasis y resecciones hepáticas

\begin{tabular}{lc}
\hline Variable & n (\% o rango) \\
\hline Quimioterapia neoadyuvante & $8(61,5)$ \\
Quimioterapia adyuvante & $12(92,3)$ \\
Ecografía intraoperatoria & $12(93,3)$ \\
Resección limitada & $12(92,3)$ \\
Segmentectomía doble & $1(7,7)$ \\
Maniobra de Pringle & $2(15,3)$ \\
Resección simultánea de colon & $7(53,8)$ \\
Nueva hepatectomía & $1(7,7)$ \\
Ligadura portal derecha & $1(7,7)$ \\
Alcoholización & $1(7,7)$ \\
Tiempo quirúrgico (minutos) & $128,46(60-220)$ \\
Pacientes con tiempo quirúrgico & $4(30)$ \\
mayor de 120 minutos & \\
Número de metástasis & $2,46(1-4)$ \\
Numero de metástasis $\geq 3$ & $6(46)$ \\
Tamaño de metástasis $(\mathrm{cm})$ & $2,56(1,8-3,5)$ \\
Pacientes con metástasis $\geq 3$ cm & $5(38,4)$ \\
Margen libre en cm & $0,86(0,5-2)$ \\
Diámetro de metástasis más margen & \\
libre en cm & $3,43(2,4-5,3)$ \\
Pacientes con diámetro de metástasis & \\
más margen libre $\geq 3$ cm & $(151,5)$ \\
Complicaciones & \\
Reingresos & \\
\hline
\end{tabular}

en promedio, antes de las 12 horas posoperatorias. La mediana de la estancia hospitalaria fue de 3,69 días, teniendo en cuenta los reingresos y que en siete casos se asoció con una resección de colon. El programa se cumplió en 85,38 \% de los casos y se consiguió la recuperación completa al egreso en seis $(46,2 \%)$.

Se midieron el diámetro de la lesión y el de la lesión más el margen, con la intención de determinar si el tamaño total de la resección tenía alguna incidencia en los resultados. También, se valoró si las cirugías con duración mayor de 120 minutos tenían repercusiones negativas. En ninguno de ellas hubo retraso en la deambulación, aumento del índice de pérdida hemática o del número de complicaciones, ni prolongación de la estancia hospitalaria (tabla 5).

\section{Discusión}

En los últimos años se ha observado un incremento en el uso de programas ERAS en cirugía abdominal mayor. La mayoría de los trabajos muestra que en cirugías de 
TABLA 3.

Pérdidas hemáticas

\begin{tabular}{lc}
\hline Volumen de sangrado $(\mathbf{m l})$ & $\mathbf{n}(\%)$ \\
\hline 0 a 100 & $8(61,5)$ \\
0 a 300 & $2(15,4)$ \\
300 a 600 & $1(7,7)$ \\
600 a 1.000 & $1(7,7)$ \\
Más de 1.000 & $1(7,7)$ \\
Total & $13(100)$ \\
\hline
\end{tabular}

TABLA 4.

Parámetros del programa implementado

\begin{tabular}{lc}
\hline Variable & n (\% o rango) \\
\hline Drenaje de cavidad & $2(15,4)$ \\
Catéter epidural & $2(15,4)$ \\
Morfina intratecal & $11(84,6)$ \\
Retiro de hidroterapia en horas & $29,54(12-72)$ \\
Retiro de sonda vesical en horas & $19,08(6-48)$ \\
Retiro de drenaje en horas & $20(16-24)$ \\
Inicio de deambulación en horas & $11,69(6-24)$ \\
Estancia hospitalaria & $3,69(2-6)$ \\
(contando reingresos) en días & \\
Cumplimiento del tratamiento & $85,38(70-100)$ \\
Casos de recuperación completa & $6(46,2)$ \\
\hline
\end{tabular}

TABla 5.

Factores pronósticos evaluados

\begin{tabular}{lcccc}
\hline Variable & $\begin{array}{c}\text { Deambu- } \\
\text { lación }\end{array}$ & $\begin{array}{c}\text { Pérdidas } \\
\text { hemáticas }\end{array}$ & $\begin{array}{c}\text { Complica- } \\
\text { ciones }\end{array}$ & $\begin{array}{c}\text { Estancia } \\
\text { Hospitalaria }\end{array}$ \\
\hline $\begin{array}{l}\text { Más de } 3 \\
\text { metástasis }\end{array}$ & 0,824 & 0,192 & 1,00 & 0,762 \\
$\begin{array}{l}\text { Tamaño mayor } \\
\text { de } 3 \mathrm{~cm}\end{array}$ & 0,288 & 0,128 & 1,00 & 0,816 \\
$\begin{array}{l}\text { Metástasis y } \\
\text { margen mayor } \\
\text { de } 3 \text { cm }\end{array}$ & 0,495 & 0,487 & 0,487 & 0,535 \\
$\begin{array}{l}\text { Tiempo de } \\
\text { cirugía mayor } \\
\text { de } 120 \text { minutos }\end{array}$ & 0,689 & 1,00 & 1,00 & 0,120 \\
$\begin{array}{l}\text { Nueva } \\
\text { hepatectomía }\end{array}$ & 0,782 & 0,154 & 0,154 & 0,089 \\
$\begin{array}{l}\text { Resección } \\
\text { simultánea de } \\
\text { colon }\end{array}$ & 0,374 & 0,192 & 1,00 & 0,034 \\
$\begin{array}{l}\text { Tabaquismo } \\
\text { Alcoholismo }\end{array}$ & 0,749 & 1,00 & 1,00 & 0,744 \\
Obesidad & 0,782 & 1,00 & 1,00 & 0,671 \\
\begin{tabular}{l} 
Ejercicio físico \\
\hline
\end{tabular} & 0,940 & 0,128 & 1,00 & 0,245 \\
\hline
\end{tabular}

colon, estómago, páncreas y esófago, estos protocolos de manejo disminuyen la estancia hospitalaria, aumentan el bienestar del paciente y disminuyen la morbilidad. La mayoría coincide en reportar una franca disminución de la estancia hospitalaria ${ }^{7-11}$. Hasta el 2016, existían pocas publicaciones sobre programas ERAS usados en resecciones hepáticas ${ }^{16}$. En ese año, en nuestra institución se inició su implementación en resecciones menores abiertas por metástasis de origen colorrectal, con resección simultánea del tumor primario o sin ella.

Fundamentalmente, con los protocolos ERAS se pretende atenuar algunos factores que favorecen la inflamación y disminuyen la reacción inmunitaria, como la agresión de la laparotomía, la pérdida hemática y la transfusión de hemoderivados, el uso moderado de opioides y la alimentación perioperatoria. Algunos de ellos actuarían disminuyendo la toxicidad de las células asesinas naturales (natural killer) y suprimiendo la inmunocompetencia de los linfocitos $\mathrm{T}$ ayudadores (T helper), mientras que el ayuno perioperatorio disminuye la captación de glucosa mediada por la insulina en el músculo esquelético y el tejido adiposo, lo cual favorece la hiperglucemia posoperatoria resistente a la insulina y sus consecuencias ${ }^{21}$.

La mayoría de los protocolos para hepatectomías, si bien presentan puntos comunes, suelen diferir unos de otros ${ }^{17}$. Nosotros hemos adaptado uno a la realidad de nuestros hospitales públicos. Al evaluar los datos obtenidos, se notó una disminución de la estancia hospitalaria de alrededor de tres días, lo que coincide con la mayoría de los reportes, aún teniendo en cuenta los reingresos ${ }^{7-11,21}$. Si bien se han abordado solo hepatectomías menores, más del $50 \%$ de los casos se asociaron con resecciones de colon. Solo en dos pacientes se presentaron complicaciones. En uno de ellos, sometido a hepatectomía por tercera vez y con un sangrado de alrededor de $1.000 \mathrm{ml}$, se presentó un síndrome anémico. Si bien se trata de un caso aislado, sugiere que las hepatectomías repetidas pueden ser una limitación para el uso de un programa ERAS. En la investigación de factores que pudieran favorecer retardos en la deambulación, o aumento de las pérdidas hemáticas, de las complicaciones o de la estancia hospitalaria, no se encontró ninguno que tuviera una incidencia significativa, aunque estos resultados iniciales deben tomarse con cuidado ya que la población estudiada fue solo de 12 pacientes. 
En una revisión sistemática de 23 puntos de un programa ERAS, se halló que cinco de ellos tenían un fuerte nivel de 'evidencia' clínica y, por ende, un alto nivel de recomendación ${ }^{22}$. Los puntos evaluados fueron los siguientes: 1) corrección de la malnutrición preoperatoria, considerando que un índice de masa corporal (IMC) inferior a 18 es una indicación para mejorar el estado nutricional antes de practicar la cirugía ${ }^{23,24}$; 2) evitación de la intubación nasogástrica en el posoperatorio inmediato, porque favorecería el reflujo y la broncoaspiración ${ }^{25,26} ; 3$ ) inicio de la alimentación en las primeras 24 horas de posoperatorio ${ }^{27}$; 4) prevención del retardo de la evacuación gástrica, y 5) estimulación de los movimientos intestinales.

En el protocolo evaluado, se incluyeron estos cinco puntos. No obstante, en cuanto a evitar el retardo de la evacuación gástrica, no se utilizó el colgajo epiploico (omental flap) que se recomienda principalmente en resecciones del colon izquierdo; se utilizaron procinéticos con moderación. Por otra parte, el tratar de obviar los drenajes obliga a una excelente hemostasia y control de las fugas biliares, para evitar colecciones importantes $\mathrm{y}$, por ende, la parálisis del tubo digestivo.

Con respecto al estímulo de la motilidad intestinal, no acostumbramos los enemas sino el tacto rectal; en casos de resección de colon y anastomosis primaria, cuando no es efectivo el tacto rectal, se coloca una sonda rectal durante algunas horas. En idéntico sentido, el inicio de la ingestión oral dentro de las primeras horas estimula la aparición de la motilidad intestinal.

En la misma revisión sistemática, mencionan otros puntos con recomendación fuerte, aunque algunos de ellos con menor nivel de 'evidencia' clínica. Entre estos puntos, vale la pena destacar el consejo preoperatorio; se ha notado que aquellos pacientes en los que el conocimiento exacto de lo que se le realizaría y de lo que se espera de ellos, favorece el cumplimiento del protocolo.

Dos aspectos importantes en el desarrollo de estos protocolos son el porcentaje de su observancia y la recuperación total en el momento del egreso.

La observancia del protocolo ERAS empleado en nuestra institución ha sido del $85 \%$, porcentaje más alto que el referido por algunos autores ${ }^{17}$. Hay coincidencia en que el porcentaje de observancia fue disminuyendo a medida que se acercaba el egreso; en la presente serie, el mayor porcentaje de cumplimiento se observó en el preoperatorio y, el más bajo, en el posoperatorio. En este último periodo, los puntos más difíciles de implementar fueron el inicio precoz de la dieta y la deambulación.

En el presente grupo de estudio, se había logrado la recuperación total en el momento del egreso en $46 \%$ de los casos; lo más difícil de controlar fue el dolor de la herida quirúrgica, como también, lograr la deambulación y las deposiciones adecuadas antes del alta. Estos resultados coinciden con los publicados por otros autores ${ }^{17}$

En conclusión, estos resultados, si bien son iniciales, muestran que en las hepatectomías menores con resecciones simultáneas de colon o sin ellas, los programas ERAS favorecen la disminución de los días de estancia hospitalaria y de los costos. La incorporación de un mayor número de casos y de hepatectomías mayores, favorecerá obtener conclusiones con mayor peso estadístico y, por ende, con un nivel de recomendación más alto.

\title{
Early experience in the application of an ERAS program in minor open hepatectomies for metatstases of colorectal cancer, with or without simultaneous colon resection
}

\begin{abstract}
Introduction: The application of fast track programs in major abdominal surgery was initially implemented in open colonic surgery with comparable results to the laparoscopic approach. The decreases in hospital stay and in hospital costs were the main advantages. Recently the application of ERAS programs to liver surgery was transferred with apparently encouraging results. The aim of this study was to evaluate the initial results obtained in a group of patients undergoing hepatectomies due to hepatic metastases with or without simultaneous colon resection.
\end{abstract}


Methods: Data from 13 minor liver resections for colorectal liver metastases with or without simultaneous resection of the primary tumor incorporated into an ERAS protocol were evaluated. Demographic data, hepatectomies, adherence to the protocol, and some factors that may affect the results of a rapid recovery, as well as what is considered full recovery at discharge, were evaluated.

Results: in the 13 resections the application of the protocol could be carried out. As a main result, a short hospital stay (3.69 days) was achieved, adherence to the protocol was high and the total recovery at discharge was $46.2 \%$.

Conclusion: the application of this protocol has been successful in terms of a frank reduction of the hospital stay. The number of patients is small but the results are encouraging.

Key words: Colonic neoplasms; neoplasm metastasis; hepatectomy; clinical protocols; learning curve; clinical evolution; cost-effectiveness evaluation.

\section{Referencias}

1. Kehlet H, Mogensen T. Hospital stay of 2 days after open sigmoidectomy with a multimodal rehabilitation programme. Br J Surg. 1999;86:227-30.

2. Kehlet H, Wilmore D. Multimodal strategies to improve surgical outcome. Am J Surg. 2002;183:630-41.

3. Gillissen F, Ament S, Maessen J, Dejong C, Dirksen C, van der Weijden T, et al. Sustainability of an Enhanced Recovery after Surgery Program (ERAS) in colonic surgery. World J Surg. 2015;39:526-33.

4. Coolsen M, van Dam R, van der Wilt A, Slim K, Lassen K, Dejong C. Systematic review and meta-analysis of enhanced recovery after pancreatic surgery with particular emphasis on pancreaticoduodenectomies. World J Surg. 2013;37:1909-18.

5. Jeong O, Park Y, Jung M, Ryu S. Compliance with guidelines of enhanced recovery after surgery in elderly patients undergoing gastrectomy. World J Surg. 2017;41:1040-6.

6. Connor S, Cross A, Sakowska M, Linscott D, Woods J. Effects of introducing an enhanced recovery after surgery programme for patients undergoing open hepatic resection. HPB (Oxford). 2013;15:294-301.

7. Varadhan KK, Neal KR, Dejong $\mathrm{CH}$, Fearon KC, Ljungqvist $\mathrm{O}$, Lobo DN. The enhanced recovery after surgery (ERAS) pathway for patients undergoing major elective open colorectal surgery: A meta-analysis of randomized controlled trials. Clin Nutr. 2010; 29:434-40.

8. Muller S, Zalunardo MP, Hubner M, Clavien PA, Demartines N. A fast-track program reduces complications and length of hospital stay after open colonic surgery. Gastroenterology. 2009;136:842-7.

9. Šrerclová Z, Dytrych P, Marvan J, Nova K, Hankeova Z, Ryska $\mathrm{O}$, et al. Fast-track in open intestinal surgery: Prospective randomized study (Clinical Trials Gov Identifier No. NCT00123456). Clin Nutr. 2009;28:618-24.
10. Spanjersberg WR, Reurings J, Keus F, van Laarhoven C. Fast track surgery versus conventional recovery strategies for colorectal surgery. Cochrane Database Syst Rev. 2011;(2):CD007635 doi: 10.1002/14651558

11. Lv L, Shao YF, Zhou YB. The enhanced recovery after surgery (ERAS) pathway for patients undergoing colorectal surgery: An update of meta-analysis of randomized controlled trials. Int J Colorectal Dis. 2012;27:1549-54.

12. Anderson A, McNaught C, MacFie J, Tring I, Barker P, Mitchell C. Randomized clinical trial of multimodal optimization and standard perioperative surgical care. Br J Surg. 2003;90: 1497-504.

13. Gatt M, Anderson A, Reddy B, Hayward-Sampson P, Tring I, MacFie J. Randomized clinical trial of multimodal optimization of surgical care in patients undergoing major colonic resection. Br J Surg. 2005;92:1354-62.

14. Eskicioglu C, Forbes SS, Aarts M-A, Okrainec A, McLeod RS. Enhanced recovery after surgery (ERAS) programs for patients having colorectal surgery: A meta-analysis of randomized trials. J Gastrointest Surg. 2009;13:2321-9.

15. Zhuang CL, Ye XZ, Zhang XD, Chen BC, Yu Z. Enhanced recovery after surgery programs versus traditional care for colorectal surgery: A meta-analysis of randomized controlled trials. Dis Colon Rectum. 2013;56:667-78.

16. Page A, Ejaz A, Spolverato G, Zavadsky T, Grant M, Galante DJ, et al. Enhanced recovery after surgery protocols for open hepatectomy--physiology, immunomodulation and implementation. J Gastrointest Surg. 2015;19:387-99.

17. Wong-Lun-Hing EM, van Dam RM, Heijnen LA, Busch OR, Terkivatan T, van Hillegersberg R, et al. Is current perioperative practice in hepatic surgery based on enhanced recovery after surgery (ERAS) principles? World J Surg. 2014;38: $1127-40$. 
18. Strasberg SM, Phillips C. Use and dissemination of the brisbane2000 nomenclature of liver anatomy and resections. Ann Surg. 2013;257:377-82.

19. Koch M, Garden OJ, Padbury R, Rahbari NN, Adam R, Capussotti L, et al. Bile leakage after hepatobiliary and pancreatic surgery: A definition and grading of severity by the International Study Group of Liver Surgery. Surgery. 2011;149:680-8.

20. Dindo D, Demartines N, Clavien PA. Classification of surgical complications: A new proposal with evaluation in a cohort of 6336 patients and results of a survey. Ann Surg. 2004;240: 205-13.

21. Nari G, Molina L, Gil F, Viotto L, Layún J, Mariot D, et al. Enhanced Recovery after Surgery (ERAS) in open liver resections for hepatic colorectal metastasis. Initial experience. Rev Argent Cirug. 2016;108:9-13.

22. Melloul E, Hubner M, Scott M, Snowden C, Prentis J, Dejong $\mathrm{C}$, et al. Guidelines for perioperative care for liver surgery: Enhanced recovery after surgery (ERAS) society recommendations. World J Surg. 2016;40:2425-40.

23. Weimann A, Braga M, Harsanyi L, Laviano A, Ljungqvist O, Soeters $\mathrm{P}$, et al. ESPEN guidelines on enteral nutrition: Surgery including organ transplantation. Clin Nutr. 2006;25:224-44.
24. Schindler K, Perincka E, Laviano A, Howard P, Schultz T, Bauer $\mathrm{P}$, et al. How nutritional risk is assessed and managed in European hospitals: A survey of 21,007 patients findings from 2007-2008 cross-sectional nutrition Day survey. Clin Nutr. 2010;29:552-9.

25. Nelson R, Edwards S, Tse B. Prophylactic nasogastric decompression after abdominal surgery. Cochrane Database Syst Rev. 2007;(3):CD004929.

26. Pessaux P, Regimbeau J, Dondero F, Plasse M, Mantz J, Belghiti J. Randomized clinical trial evaluating the need for routin nasogastric decompression after elective hepatic resection. $\mathrm{Br}$ J Surg. 2007;94:297-303.

27. Lassen K, Kjaeve J, Fetveit T, Trano G, Sigurdsson H, Horn A, et al. Allowing normal food at will after major upper gastrointestinal surgery does not increase morbidity: A randomized multicenter trial. Ann Surg. 2008;247:721-9.

Correspondencia: Gustavo Adrian Nari, MD Correo eletrónico: gusnari@hotmail.com Córdoba, Argentina 